\title{
EN TORNO A LA ALTERNANCIA MODAL DESPUÉS DE EL HECHO DE QUE
}

\author{
Wiaczesław Nowikow \\ Universidad de Łódź \\ http://dx.doi.org/10.18778/8220-201-4.22
}

\section{Resumen}

En el artículo se examina la selección modal de los modos indicativo y subjuntivo en las construcciones con el hecho de que en el español actual. El análisis se lleva a cabo a base de los datos procedentes del Corpus del Español del Siglo XXI (CORPES) de la Real Academia Española girando en torno a dos problemas: 1. las frecuencias globales de las formas de los modos en cuestión (frecuencias absoluta y normalizada); 2. la comparación de la elección de los dos modos indicativo y subjuntivo en diferentes zonas geográficas y países (10 zonas y 23 países). El análisis del material (157 casos de 15 formas modotemporales de los verbos ser, estar y tener de los años 2001-2016) demostró el predominio global del modo subjuntivo. No obstante, no en todas las zonas la situación es la misma (p.ej., España y la zona Andina), lo que ponen de manifiesto los comentarios de los resultados presentados en el trabajo.

Palabras clave: El hecho de que, indicativo y subjuntivo, selección modal, frecuencias absoluta y normalizada, repartición geográfica.

\section{1.}

\section{Introducción}

Como es sabido, en el español moderno las construcciones con el hecho de que admiten tanto el modo indicativo (IND) como el subjuntivo (SUBJ). Dicha alternancia ha llamado la atención en varias ocasiones sobre todo por el carácter factitivo del 
sustantivo el hecho que se asocia, en principio, con el contenido funcional del IND. A primera vista, esta asociación se presenta como algo natural al tener en cuenta que el valor gramatical del IND se define, a menudo, como [aserción], es decir, compromiso con la verdad de lo referido. Pues, para los interlocutores o para los autores de los textos y de los lectores de estos, un acontecimiento o un rasgo presentados mediante la palabra $\mathrm{el}$ hecho suelen ser percibidos e interpretados como verdaderos y, en este sentido, factitivos. Este último concepto, llamado también fáctico, factual o factible (se remontan al lat. factum), al significar 'perteneciente o relativo a hechos', permite presentar la referencia como algo basado en hechos, en oposición a lo teórico o a lo imaginario.

No obstante, actualmente (y en realidad desde hace muchísimos años) el empleo del SUBJ después de el hecho de que es un fenómeno muy difundido y, como se indica a veces, desde el punto de vista estilístico, el uso de este último modo rebasa el del IND en el habla más cuidada y controlada (Fente, Fernández, Feijóo, 1975: 54-55; Nowikow, 2017: 85). De modo que en la construcción en cuestión en el español de hoy se dan los dos modos señalados supra, p. ej.:

(1) El hecho de que escribió (IND) el libro impresionado por la pérdida de su madre explica la presencia obsesiva de la muerte en varias páginas de la obra.

(2) El hecho de que el desempleo sea (SUBJ) una prioridad para las autoridades explica la presencia de muchas estadísticas al respecto en los documentos gubernamentales.

Dada la posibilidad de la doble selección modal en el núcleo predicativo (NP) después de el hecho de que, hemos decidido llevar a cabo una pequeña investigación sobre la difusión del fenómeno en cuestión en el español actual, haciéndolo mediante el análisis del material procedente del Corpus del Español del Siglo XXI (CORPES XXI, Versión beta [0.91], Consultas: 24-25/02/2019) de los años 2001-2016. Antes de pasar a los comentarios sobre las pautas y los resultados de la investigación, vamos a hacer una breve introducción a la problemática relacionada 
con las características sintáctico-semánticas de la construcción el hecho de que + NP [IND / SUBJ], puesto que entre estas últimas, al parecer, hay ciertos factores que influyen en la selección modal.

\section{2.}

\section{Algunas propiedades sintáctico- semánticas de la construcción con el hecho de que y los factores que influyen en la selección modal}

Como es sabido (Pamies / Nowikow, 2015: 65-67), hay polémicas sobre el estatus sintáctico de la construcción el hecho de que + NP [IND / SUBJ], considerada esta como cláusula preposicional sustantiva o relativa. Esto ocurre porque, por un lado, la secuencia que sigue al sustantivo el hecho se introduce mediante una preposición (de) y complementa al nombre antepuesto, lo que quiere decir que aquella tiene función adjetival. Por otro lado, la secuencia el hecho de que + NP [IND / SUBJ] puede desempeñar el papel del núcleo nominal (NN) respecto al sintagma pospuesto que contiene un núcleo predicativo (NP). Por ejemplo en la oración

(3) El hecho de que hayas pagado tus impuestos no te da derecho a nada

la secuencia el hecho de que hayas pagado tus impuestos al establecer una relación de tipo adjetival entre sus componentes, podría equivaler sintácticamente a el/un hecho muy importante, significativo, actual, etc. Lo mismo se refiere a otros sustantivos que pueden estar en posición de argumento, p. ej., la idea de que, la razón de que, etc. Al mismo tiempo la relación entre el hecho de que hayas pagado tus impuestos y no te da derecho a nada corresponde a la de entre un sintagma nominal (SN) y otro verbal (SV). En otras palabras, la relación sintáctica establecida podría equivaler a la que se da entre los NN y NP en cláusulas tales como, p. ej., El dinero/ el poder no te da derecho a nada. Cabe añadir que 
la construcción en cuestión puede ser nominalizada a través de la elisión del NN regente y de la preposición de:

(4) El que hayas pagado tus impuestos no te da derecho a nada.

Parece que la interpretación sintáctica "adjetival" (relativa) se defiende mejor en los casos cuando el hecho de que se encuentra en posición inicial, es decir, encabeza toda la oración y se antepone a los demás elementos de esta última. Observemos que en tales distribuciones la secuencia en cuestión suele formar parte de la cláusula principal. En cambio, cuando a el hecho de que le antecede el NP regente (subordinante) parece ser mas posible la interpretación "sustantiva" (nominal), p. ej.:

(5) Admito (el hecho de) que no puedes ayudarme.

(6) Me da mucha pena (el hecho de) que no puedas auydarme.

Algunos autores (Delbecque / Lamiroy, 1999: 1967; Leonetti, 1999: 2085) consideran tales construcciones como subordinadas sustantivas enunciativas, donde el hecho de es un elemento sintáctico opcional que actúa como correlato del contenido semántico de la cláusula subordinada. Al mismo tiempo, parece que el hecho de, hasta cierto punto, debilita la subordinación matizando la relación sintáctica entre las dos cláusulas de conexión apositiva (véase, a este respecto, p. ej., Delbecque / Lamiroy, 1999: 1970; Leonetti, 1999: 2100). En opinión de Delbecque y Lamiroy (ibíd.), el hecho de que no es opcional con los predicados en cuyo caso el empleo de que se ve problemático, p. ej., lo excusa ? que (cfr. lo excusa el hecho de que...).

En cambio, desde el punto de vista semántico, el hecho de puede ser considerado como una marca epistémica que, como hemos señalado supra, sugiere el carácter supuestamente factitivo del contenido de la secuencia conectada sintácticamente mediante subordinación / aposición. Algunos autores, como Leonetti (1999: 2101), subrayan la relación especial de el hecho de que con los predicados factitivos señalando que estos al escoger el modo SUBJ, presuponen la verdad de la subordinada. No obstante, como demuestran los ejemplos (5) y (6), el hecho de que se construye con los dos modos. Por otro lado, cabe subrayar que en el caso de las 
cláusulas subordinadas sustantivas con el hecho de que la selección modal IND / SUBJ a menudo depende de las características semánticas del NP regente. Los llamados verbos factitivos fucionan en varias ocasiones como predicados que en primer lugar valoran el contenido de la cláusula subordinada, lo que explica la selección habitual del SUBJ.

Sin embargo, aunque las propiedades semánticas del NP regente son muy importantes para la selección modal, no son las únicas que deciden sobre la elección del modo verbal. Hay por lo menos dos factores más que influyen en la selección del modo SUBJ en la cláusula subordinada. Uno se ve relacionado con el concepto de irrelevancia informativa mientras que otro con el de negación.

La primera noción se refiere a la llamada anáfora textual y, en concreto, a las evocaciones intertextuales que marcan irrelevancia informativa (cfr. Nowikow, 1999: 77 y 2001: 141-143) de tipo

(7) El hecho de que seas mi hermano no significa que siempre tengas razón.

El fenómeno se observa también en otras construcciones donde se transmiten las informaciones compartidas por los interlocutores, por ejemplo, en las subordinadas concesivas con aunque (véase Nowikow, 2017: 111-112) o en las sustantivas con los predicados que presuponen y / o valoran el contenido de la clásula subordinada (me encanta, extraña, molesta, etc., cfr. Nowikow, 2017: 62-64). Sin entrar en los detalles, subrayemos que se trata de los casos cuando a menudo resulta difícil cuestionar la factualidad de la acción referida. En tales distribuciones el papel del SUBJ es diferente: al referirse a los sucesos que se caracterizan por poca importancia informativa, este modo desempeña en dichas construcciones una función pragmático-informativo-textual que consiste en la marcación de los contenidos informativamente irrelevantes.

En cambio, la negación, igual que la irrelevancia informativa, es un factor muy importante que con frecuencia influye en la selección del modo subjuntivo. Así ya hace años Fente, Fernández, Feijóo (1975: 55) señalaban que en varios casos con el hecho de que se niega que el hecho referido "sea un motivo válido para el hablante", por ejemplo: 
(8) El hecho de que no haya venido no debe hacerte pensar que esté enfadado (el ejemplo de Fente, Fernández, Feijóo, ibid.).

Finalmente, cabe recordar también un factor de índole estilística. Como ya hemos indicado supra, en algunos trabajos (Fente, Fernández, Feijóo, 1975: 54, Nowikow, 2017: 85) se señala que el SUBJ prevalece en la expresión más cuidada y controlada, lo que significa que dicho modo predomina en el estilo literario.

En resumen, en la construcción con el hecho de que se observa la doble selección modal IND / SUBJ. Podemos admitir que la selección del IND se ve relacionada, principalmente, con la factualidad expresada por el sustantivo el hecho, lo que ocurre sobre todo cuando el hablante quiere constatar y subrayar el carácter factitivo de los hechos referidos. Este factor semántico-discursivo es muy importante, pero no es el único que decide sobre la elección del modo verbal. En realidad, los parámetros que favorecen y condicionan el empleo del SUBJ son más numerosos. A estos se refieren la valoración y la negación de los acontecimientos denotados en la cláusula subordinada así como la posible irrelevancia informativa de estos últimos. Además, se añade el factor estilístico que a menudo contribuye a la selección del modo subjuntivo.

3.

La selección modal IND / SUBJ en la construcciones el hecho de que + ser / estar / tener [imperfecto subj] en el CORPES XXI

\section{1.}

\section{Las características del corpus y del material analizado}

Como hemos anticipado en la Introducción presentada supra, examinamos los datos procedentes del Corpus del Español del Siglo XXI (CORPES) de la Real Academia Española. La versión actual 0.91 existe desde diciembre de 2018 y está formada por unos 
285000 documentos que cuentan con algo más de 286 millones de formas. Los datos proceden de textos de ficción (novelas, obras de teatro, etc.) y de no ficción (salud, política, artes, tecnología, ciencias sociales). En torno a 150 millones formas provienen de prensa, entrevistas digitales y blogs. Por otro lado, el CORPES XXI sigue siendo un recurso en construcción. Por ejemplo, la versión 0.91 con respecto a la de 2016 incorpora aproximadamente 30 millones de formas ortográficas más.

Nuestro análisis incluye las formas modotemporales de tres verbos: ser, estar y tener. Los usos del primero se refieren a los años 2001-2016 mientras que de los dos últimos atañen al periodo 2001-2015. En concreto, el material examinado se compone de 15 formas gramaticales de $1^{\text {a }}$ y $3^{\text {a }}$ pers. sing. (5 formas de cada uno de los verbos): 9 de indicativo (ser: era, fui / fue; estar: estaba, estuve / estuvo; tener: tenía, tuve / tuvo) y 6 de subjuntivo (ser:fuera / fuese; estar: estuviera / estuviese; tener: tuviera / tuviese). La elección de los verbos no fue casual. La versión 0.91 del CORPES al descontar signos ortográficos (puntos, comas, etc.), contiene unos 280 millones de elementos gramaticales. Cabe subrayar que los textos fueron anotados morfosintácticamente y lematizados, lo que permitió obtener estadísticas que se referían tanto a elementos gramaticales como a lemas. Por ejemplo, las formas gramaticales canta, cantaba, cantó, cante, cantara, cantase, etc. han sido reunidas bajo el lema cantar. Al descontar nombres propios, el CORPES XXI cuenta con 259838 lemas reunidos en los listados de frecuencia de lemas. Los verbos ser, estary tener elegidos para el análisis revelan la mayor frecuencia entre los verbos (ocupan tres primeros lugares) que se encuentran en el listado de mil lemas frecuentes. En otras palabras, dichos verbos demuestran las más altas frecuencias general y normalizada. La primera (general o absoluta), es el número de veces que determinada forma gramatical aparece en el total del corpus. No obstante, la importancia de esta cantidad depende también del tamaño del corpus. Por ejemplo, el corpus de los textos procedentes de España es considerablemente superior al de los textos de Guatemala o Nicaragua. Lógicamente, el número de los elementos gramaticales analizados depende, en gran medida, de la cantidad de los textos presentes en el corpus analizado. Por eso, hay que tener en cuenta también la 
frecuencia normalizada que permite comparar un fenómeno (o una forma) en corpus de diferentes tamaños. La frecuencia normalizada se refiere al número de veces que aparece una determinada forma por cada millón de elementos o a la expresión porcentual sobre el total de formas del corpus (p. ej., 30688,85 vs. $3,07 \%)^{1}$.

A raíz de la dimensión restringida de este artículo nos limitamos a la presentación de dos tipos de datos:

a) las cifras globales que reflejan la cantidad de las formas de indicativo y subjuntivo seleccionadas (comparación de los modos IND y SUBJ);

b) los resultados que se refieren a la elección entre los dos modos en diferentes zonas geográficas y países, que son los siguientes:

1. España;

2. Zona Andina: Bolivia, Ecuador, Perú;

3. Antillas: Cuba, Puerto Rico, República Dominicana;

4. Caribe continental: Colombia, Venezuela;

5. Zona Chilena: Chile;

6. México y Centroamérica: Costa Rica, El Salvador, Guatemala, Honduras, México, Nicaragua, Panamá;

7. Río de la Plata: Argentina, Paraguay, Uruguay;

8. Estados Unidos;

9. Filipinas;

10. Guinea Ecuatorial.

\section{2.}

\section{La repartición global entre modos y tiempos}

En total registramos 157 formas verbales construidas con el hecho de que: 97 de ser, 32 de estar y 28 de tener. Estas se presentan de la siguiente manera:

1 Todos los datos y comentarios sobre las frecuencias absoluta y normalizada provienen de la página de la RAE (CORPES XXI; estadística) y de la comunicación personal del prof. dr Guillermo Rojo, académico responsable del proyecto Corpus del español del siglo XXI (CORPES). 
Cuadro 1. Frecuencia absoluta de ser, estar y tener en la selección de los modos IND y SUBJ

\begin{tabular}{l|c|c|c}
\multicolumn{1}{c|}{ VERBOS } & IND & SUBJ & TOTAL \\
Ser & 34 & 63 & 97 \\
\hline Estar & 13 & 19 & 32 \\
\hline Tener & 10 & 18 & 28 \\
\hline Total & $57(36,3 \%)$ & $100(63,7 \%)$ & 157 \\
\hline
\end{tabular}

Se ve un claro predominio del SUBJ cuyo porcentaje alcanza el 63,7\%, lo que constituye casi el doble de las formas del IND (57 formas; el 36,3\%).

En cuanto a la repartición de los tiempos dentro de los modos indicativo (copretérito vs. pretérito) y subjuntivo (copretérito: cantara vs. cantase), esta ha sido la siguiente:

Cuadro 2. Frecuencia absoluta de ser, estar y tener en los tiempos de IND: copretérito (imperfecto) vs. pretérito

\begin{tabular}{l|c|c|c} 
IND & $\begin{array}{c}\text { COPRETÉRITO } \\
\text { (IMPERF.) }\end{array}$ & PRETÉRITO & TOTAL \\
Era & 20 & - & 20 \\
\hline Fue & - & 14 & 9 \\
\hline Estaba & 12 & - & 12 \\
\hline Estuvo & - & 1 & 1 \\
\hline Tenía & 9 & - & 9 \\
\hline Tuve & - & 1 & 1 \\
\hline Total & $41(71,9 \%)$ & $16(28,1 \%)$ & 57 \\
\hline
\end{tabular}


Cuadro 3. Frecuencia absoluta de ser, estar y tener en el copretérito (imperfecto) del SUBJ: cantara vs. cantase

\begin{tabular}{lcccc}
\multicolumn{1}{c}{ SUBJ } & CANTARA & CANTASE & TOTAL \\
Fuera & 55 & - & 55 \\
\hline Fuese & - & 8 & 8 \\
\hline Estuviera & 16 & - & 16 \\
\hline Estuviese & - & 3 & 3 \\
\hline Tuviera & 17 & - & 17 \\
\hline Tuviese & - & 1 & 1 \\
\hline Total & $88(88 \%)$ & $12(12 \%)$ & 100 \\
\hline
\end{tabular}

Con respecto al IND, los datos demuestran que prevalecen con gran ventaja las formas del copretérito (cantaba): 41 (71,9\%) frente a las $16(28,1 \%)$ del pretérito (cantó), lo que podría estar relacionado con el factor diafásico-discursivo: la mayoría de los textos del corpus pertenece al bloque "ficción" (novelas, relatos, etc.) que se caracteriza por un fuerte componente narrativo. No se han registrado las formas fui, estuve y tuvo.

En cambio, en el copretérito (imperfecto) de SUBJ predomina con una ventaja aún más grande la forma en -ra: 88 casos (88\%) frente a solo 12 casos de la forma en $-s e(12 \%)$. Cabe subrayar que de 12 formas de tipo cantase 10 se dieron en los textos españoles (del total de 51 formas subjuntivas peninsulares), lo que quiere decir que en la Península las formas en -se constituyen un $20 \%$. Esto significa que en las fuentes peninsulares la frecuencia de cantara ha sido cinco veces más alta que la de cantase. En los textos hispanoamericanos la forma en -se apareció solo dos veces (el total de las formas subjuntivas: 49) siendo su porcentaje muy bajo: en torno a un $4 \%$. Las dos formas hispanoamericanas se dieron en el verbo ser (fuese), una en Paraguay y otra, en Venezuela:

(9) En esta época ya existían algunos sistemas de búsqueda documental que utilizaban internet, como los llamados WAIS y Gopher, pero la simplicidad de la web y el hecho de que fuese accesible de forma gratuita permitieron que esta herramienta fuese desarrollada y adoptada inmediatamente por la sociedad ("El mundo celebra los veinte 
años de la web libre, accesible y gratuita", $A B C$ Color, Asunción, abc.com.py, 2013-04-30. Clasificación CORPES: Año: 2013. Medio: Escrito. Bloque: No ficción. Soporte: Prensa. Tema: Ciencia y tecnología. País: Paraguay. Zona: Río de la Plata. Tipología: Noticia).

(10) Apenas apareció en la televisión como jefe de la intentona de golpe de Estado, su imagen tocó muy hondo en el inconsciente colectivo venezolano. Como señala la escritora y psicoanalista Ana Teresa Torres, "el hecho de que fuese un militar golpista pudiera haber generado escrúpulos en algunas mentes civilistas, pero no en el grueso de la población. Ser golpista significaba que se había jugado la vida por defender la patria,... (Caproles M., Axel, Las fantasías de Juan Bimba: Mitos que nos dominan, estereotipos que nos confunden, Caracas: Taurus, 2011. Clasificación CORPES: Año: 2011. Medio: Escrito. Bloque: No ficción. Soporte: Libro. Tema: Ciencias sociales, creencias y pensamiento. País: Venezuela. Zona: Caribe continental. Tipología: Académico).

Cabe señalar que desde la perspectiva diafásica los dos ejemplos pertenecen a la minoría, es decir, se dan en los textos con los parámetros de no ficción, libro / prensa, ciencia y tecnología / ciencias sociales, noticia / académico. El caso paraguayo se encuentra en un texto periodístico y no se sabe la procedencia del autor de la noticia. En cambio, en el ejemplo venezolano cantase forma parte de un fragmento donde se cita a otra persona.

De modo que se confirma una vez más la escasa difusión de cantase en América y la relativa importancia (eso sí, mucho menor que la de cantara) de esta forma en España.

\section{3.}

\section{La selección modal IND / SUBJ en diferentes zonas geográficas y países}

La repartición diatópica del empleo de los modos IND y SUBJ en la construcción con el hecho de que se ve más complicada que las selecciones modal y temporal comentadas en el párrafo anterior. 
Dado que los tamaños de los corpus de diversos países y zonas son muy diferentes (véase supra), decidimos basar nuestros análisis no solo en la frecuencia absoluta sino también en la frecuencia normalizada, siendo esta última, a veces, más importante que la primera. Si no hubiésemos tenido en cuenta el parámetro de frecuencia normalizada, casi en todas las distribuciones el primer lugar lo habría ocupado España (este es, por ejemplo, el caso de las formas del verbo ser), puesto que el corpus de los textos procedentes de esta última supera considerablemente los corpus de los textos hispanoamericanos. De ahí la alta frecuencia absoluta de las formas peninsulares. Al tomar en consideración la proporción entre el tamaño del corpus y la cantidad de las formas registradas, los usos españoles perdieron, por lo menos en algunos casos, su predominio numérico-estadístico. Pasemos a la presentación de los resultados mediante los cuadros que reflejan la repartición de las formas verbales de indicativo y subjuntivo entre zonas y países. Indicamos, donde resulta posible hacerlo, tres zonas y / o países con la frecuencia normalizada más alta. Señalamos en primer lugar la frecuencia normalizada (Fnorm.) seguida, después de una barra oblicua, por frecuencia absoluta (Freq), por ejemplo, Fnorm. / Freq: 0,09 / 2; 0,15 / 1; 0,32 / 29, etc.:

Cuadro 4. Repartición diatópica de las formas del verbo ser

\section{FORMA VERBAL \\ ZONA: FNORM. / FREQ \\ PAIS: FNORM. / FREQ}

\begin{tabular}{|c|c|c|}
\hline 1 & 2 & 3 \\
\hline \multirow{3}{*}{ Era } & Antillas: 0,15 / 3 & Puerto Rico: 0,25 / 1 \\
\hline & Chilena: 0,11 / 2 & Bolivia: 0,18 / 1 \\
\hline & Andina: 0,09 / 2 & Rep. Dominicana: 0,16 / 1 \\
\hline \multirow{3}{*}{ Fue } & Río de la Plata: 0,10 / 4 & El Salvador: 0,24 / 1 \\
\hline & México / Centroam.: 0,07 / 4 & Paraguay: 0,15 / 1 \\
\hline & Chilena: 0,05 / 1 & Uruguay: 0,13 / 1 \\
\hline \multirow{3}{*}{ Fuera } & Antillas: $0,40 / 8$ & Cuba: 0,60 / 6 \\
\hline & España: 0,32 / 29 & Panamá: 0,42 / 1 \\
\hline & Río de la Plata: 0,15 / 6 & España: 0,32 / 29 \\
\hline
\end{tabular}




\begin{tabular}{lll}
\hline & \multicolumn{1}{c}{2} & \multicolumn{1}{c}{3} \\
\hline \multirow{3}{*}{ Fuese } & España: 0,06 / 6 & Paraguay: 0,15 / 1 \\
& Caribe cont.: $0,02 / 1$ & Venezuela: 0,07 / 1 \\
& Río de la Plata: $0,02 / 1$ & España: 0,06 / 6 \\
\hline
\end{tabular}

Cuadro 5. Repartición diatópica de las formas del verbo estar

\begin{tabular}{|c|c|c|}
\hline FORMA VERBAL & ZONA: FNORM. / FREQ & PAÍS: F.NORM./ FREQ \\
\hline Estaba & $\begin{array}{l}\text { México / Centroam.: 0,11 / } 6 \\
\text { Río de la Plata: 0,07 / } 3 \\
\text { España: 0,01 / } 1\end{array}$ & $\begin{array}{l}\text { Honduras: 0,49 / } 2 \\
\text { Puerto Rico: 0,25 / } 1 \\
\text { El Salvador: 0,24 / } 1\end{array}$ \\
\hline Estuvo & Andina: $0,04 / 1$ & Bolivia: 0,18 / 1 \\
\hline Estuviera & $\begin{array}{l}\text { Estados Unidos: 0,28 / } 1 \\
\text { Chilena: 0,17 / } 3 \\
\text { Río de la Plata: 0,12 / } 5\end{array}$ & $\begin{array}{l}\text { Estados Unidos: 0,28 / } 1 \\
\text { Chile: } 0,17 \text { / } 3 \\
\text { Argentina: 0,15 / } 5\end{array}$ \\
\hline Estuviese & España: 0,03 / 3 & España: 0,03 / 3 \\
\hline
\end{tabular}

Cuadro 6. Repartición diatópica de las formas del verbo tener

\begin{tabular}{|c|c|c|}
\hline FORMA VERBAL & ZONA: FNORM. / FREQ & PAIS: F.NORM. / FREQ \\
\hline Tenía & $\begin{array}{l}\text { España: } 0.05 \text { / } 5 \\
\text { Antillas: 0,05 / } 1 \\
\text { Andina: 0,04 / } 1\end{array}$ & $\begin{array}{l}\text { Puerto Rico: 0,25 / } 1 \\
\text { Ecuador: 0,14 / } 1 \\
\text { España: 0,05 / } 5\end{array}$ \\
\hline Tuve & Andina: 0.04 / 1 & Perú: 0,10 / 1 \\
\hline Tuviera & $\begin{array}{l}\text { México / Centroam.: 0,09 / } 5 \\
\text { España: 0,08 / } 8 \\
\text { Río de la Plata: 0,07 / } 3\end{array}$ & $\begin{array}{l}\text { Paraguay: 0,31 / } 2 \\
\text { Rep. Dominicana: 0,16 / } 1 \\
\text { México: 0,15 / } 5\end{array}$ \\
\hline Tuviese & España: 0,01 / 1 & España: 0,01 / 1 \\
\hline
\end{tabular}

La interpretación de estos resultados no es fácil porque 27 distribuciones del total de 54 presentadas en los cuadros se refieren a los usos que incluyen solo una forma. En cuatro distribuciones aparecen dos formas y en seis la Freq es solo de tres formas verbales. De modo que en 37 distribuciones (del total de 54) la 
frecuencia absoluta oscila entre uno y tres casos. En cambio, hay solo 8 distribuciones donde la Freq rebasa cinco formas. Subrayemos que la mayoría de estas distribuciones (cinco) se da en España.

Tampoco la frecuencia normalizada facilita la interpretación en todas las situaciones. Es significativo el empleo de la forma estuviera en cuyo caso la Fnorm. hace que la primera posición la ocupen los Estados Unidos, donde la frecuencia normalizada alcanza el 0,28 siendo más alta que en España, México, Argentina y otros países o zonas. Sin embargo, cabe subrayar que este resultado se consigue a partir de solo un empleo de estuviera. Esto quiere decir que, igual que en el caso de la frecuencia absoluta, en el de la frecuencia normalizada hay que actuar con propiedad tratando de tomar en consideración varios factores.

De modo que, para evaluar la posición del IND y del SUBJ en la selección modal desde el punto de vista de la repartición geográfica, hemos decidido hacer el cálculo basado en los datos de las zonas (en los casos de España, Chile y Estados Unidos estas coinciden con los países) que garantizan mayor cantidad de formas verbales que los de cada país por separado. Lo hemos llevado a cabo tratando de establecer una correlación en el proceso de la selección modal IND / SUBJ entre las dos frecuencias señaladas: la absoluta y la normalizada, separadas, igual que en los cuadros anteriores, por una barra oblicua:

Cuadro 7. La selección modal IND / SUBJ según la repartición geográfica por zonas

\begin{tabular}{l|c|c}
\multicolumn{1}{c|}{ ZONA } & \multicolumn{1}{c|}{$\begin{array}{c}\text { IND (SER+ESTAR+TENER): } \\
\text { FREQ / FNORM. }\end{array}$} & $\begin{array}{c}\text { SUBJ (SER+ESTAR+TENER): } \\
\text { FREQ / FNORM. }\end{array}$ \\
\hline España & $18 / 0.18$ & 3 \\
\hline Zona Andina & $6 / 0,25$ & - \\
\hline Antillas & $5 / 0,25$ & $10 / 0,50$ \\
\hline Caribe continental & $1 / 0,02$ & $3 / 0,06$ \\
\hline Zona Chilena & $3 / 0,16$ & $5 / 0,28$ \\
\hline
\end{tabular}




\begin{tabular}{lcl}
\hline \multicolumn{1}{c}{1} & 2 & 3 \\
\hline $\begin{array}{l}\text { México } \\
\text { y Centroamérica }\end{array}$ & $14 / 0,24$ & $14 / 0,24$ \\
\hline Río de la Plata & $10 / 0,24$ & $15 / 0,36$ \\
\hline Estados Unidos & - & $2 / 0,56$ \\
\hline
\end{tabular}

El ranking de la selección modal en las distintas zonas, según este cuadro y la relación Fnorm. / Freq se presenta de la siguiente manera:

IND

1. México y Centroamérica: $0,24 / 14$

2. Zona Andina: 0,25 / 6

3. Antillas: $0,25 / 5$

4. Río de la Plata: 0,24 / 10

5. España: $0,18 / 18$

6. Zona Chilena: 0,16/3

7. Caribe continental: $0,02 / 1$

8. Estados Unidos:-

\section{SUBJ}

1. Estados Unidos: $0,56 / 2$

2. España: $0,54 / 51$

3. Antillas: $0,50 / 10$

4. Río de la Plata: $0,36 / 15$

5. Zona Chilena: $0,28 / 5$

6. México y Centroamérica: $0,24 / 14$

7. Caribe continental: $0,06 / 3$

8. Zona Andina: -

No obstante, para comparar las tendencias más generales en la selección de los modos verbales, creemos conveniente tomar en consideración, en primer lugar, los resultados de las zonas que demuestran no solo la frecuencia normalizada más alta (recordemos, es la que se ve relacionada con el tamaño del corpus) sino que, al mismo tiempo, se caracterizan por una relativamente alta frecuencia absoluta (cantidad global de las formas registradas). 
Así, si tenemos en cuenta los casos cuando esta última rebasa 5 formas verbales, el ranking se presenta de la siguiente manera:

IND

1. Zona Andina: 0,25 / 6

2. México y Centroamérica: $0,24 / 14$

3. Río de la Plata: 0,24 / 10

5. España: $0,18 / 18$

\section{SUBJ}

1. España: $0,54 / 51$

2. Antillas: $0,50 / 10$

3. Río de la Plata: $0,36 / 15$

4. México y Centroamérica: 0,24 / 14

Salta a la vista la presencia de España, Río de la Plata y de México y Centroamérica en ambos listados, complementada por la Zona andina en el IND, y por las Antillas en el SUBJ. Todo ello, será objeto de comentario, junto a otros fenómenos, en las breves observaciones finales que siguen.

\section{4. \\ Observaciones finales sobre la selección modal después de el hecho de que}

Recapitulando el análisis realizado en los párrafos anteriores, cabe destacar las siguientes observaciones:

- Como hemos señalado supra, se ha registrado un predominio considerable (casi el doble) del SUBJ: 100 formas de este modo $(36,3 \%)$ frente a 57 formas del IND (63,7\%).

- Las frecuencias normalizadas del IND de las cuatro zonas principales se sitúan entre el 0,18 y el 0,25 , es decir son bastante próximas.

- No es el caso del SUBJ donde las Fnorm. de España $(0,54)$ y Antillas $(0,50)$ superan considerablemente las de Río de la Plata $(0,36)$ y México y Centroamérica $(0,24)$. Sin embargo, el 
resultado obtenido en la zona de Antillas está basado en la Freq de 10 formas, es decir, más baja que en las dos últimas zonas.

- Llama la atención el hecho de que en la zona con el mayor índice de la Fnorm. en el IND $(0,25)$, es decir, en la andina, no se registró ningún caso del SUBJ. En otras palabras, en la Zona Andina después de el hecho de que se dieron solo las formas del IND.

- En cambio, en España, zona con el índice más alto y con el predominio total del SUBJ $(0,54$ / 51), se registró la mayor diferencia entre este último modo y el IND $(0,18$ / 18). Como se ve, las frecuencias normalizada y absoluta del SUBJ son tres veces más altas que las del IND.

- Es de notar que Antillas, segunda zona con la Fnorm. del SUBJ más alta $(0,50 / 10)$, también demuestra un claro predominio con respecto al IND $(0,25 / 5)$. Las frecuencias normalizada y absoluta del SUBJ superan dos veces las del IND.

- Las demás zonas demuestran cierto equilibrio entre las frecuencias del IND y del SUBJ.

\section{Referencias bibliográficas}

Delbecque, N., Lamiroy, B. (1999), "La subordinación sustantiva. Las subordinadas enunciativas en los complementos verbales", en I. Bosque, V. Demonte (eds.), Gramática descriptiva de la Lengua Española, Vol. II, Madrid: Real Academia Española / Espasa-Calpe, 1965-2081.

Fente, R., Fernández, J., Feijóo, L.G. (1975), El subjuntivo, Madrid: S.G.E.L.

Leonetti, M. (1999), "La subordinación sustantiva. Las subordinadas enunciativas en los complementos nominales", en I. Bosque, V. Demonte (eds.), Gramática descriptiva de la Lengua Española, Vol. II, Madrid: Real Academia Española / Espasa-Calpe, 2083-2104.

Nowikow, W. (1999), "Funkcje tekstowe modalno-temporalnych form romańskich", Kwartalnik Neofilologiczny, XLI, 1-2, 73-80.

Nowikow, W. (2001), La alternancia de los modos Indicativo y Subjuntivo en las cláusulas subordinadas sustantivas (metodología 
del análisis lingüístico), Poznań: Wydawnictwo Naukowe Uniwersytetu im. Adama Mickiewicza.

Nowikow, W. (2017), "Modos verbales”, en W. Nowikow (ed.), Gramática contrastiva español-polaco, Colección "Manufactura Hispánica Lodziense”, 2, Łódź: Wydawnictwo Uniwersytetu Łódzkiego, 11-126.

Pamies Bertrán, A., Nowikow, W. (2015), Los modos verbales en español y en polaco, Colección "Manufactura Hispánica Lodziense”, 1, Łódź: Wydawnictwo Uniwersytetu Łódzkiego.

REAL ACADEMIA ESPAÑOLA: Banco de datos (CORPES XXI) [en línea]. Corpus del Español del Siglo XXI (CORPES). http:// www.rae.es, [fecha de la consulta: 24-25.02.2019]. 